\title{
Development of a Systematic Review of Public Health Interventions to Prevent Children Drowning
}

\author{
Gemma Crawford ${ }^{1}$, Justine Leavy ${ }^{1}$, Linda Portsmouth 1 , Jonine Jancey ${ }^{1}$, \\ Francene Leaversuch ${ }^{2}$, Lauren Nimmo ${ }^{3}$, Lauren Reid-Dornbusch' ${ }^{1}$ Emma Hills ${ }^{1}$ \\ ${ }^{1}$ Western Australian Centre for Health Promotion Research, Curtin University, Bentley, Australia \\ ${ }^{2}$ School of Public Health, Curtin University, Bentley, Australia \\ ${ }^{3}$ Royal Life Saving Society Western Australia Inc., Mt Claremont, Australia \\ Email: "g.crawford@curtin.edu.au
}

Received 16 January 2014; revised 17 February 2014; accepted 4 March 2014

Copyright (C) 2014 by authors and Scientific Research Publishing Inc.

This work is licensed under the Creative Commons Attribution International License (CC BY).

http://creativecommons.org/licenses/by/4.0/

(c) (i) Open Access

\begin{abstract}
Drowning is the leading cause of death from unintended injury in children globally. Drowning is preventable, and mechanisms exist which can reduce its impact, however the peer-reviewed literature to guide public health interventions is lacking. This paper describes a protocol for a review of drowning prevention interventions for children. Electronic searching will identify relevant peer-reviewed literature describing interventions to prevent child drowning worldwide. Outcome measures will include: drowning rates, water safety behaviour change, knowledge and/or attitude change, water safety policy and legislation, changes to environment and water safety skills. Quality appraisal and data extraction will be independently completed by two researchers using standardised forms recording descriptive and outcome data for each included article. Data analysis and presentation of results will occur after data have been extracted. This review will map the types of interventions being implemented to prevent drowning amongst children and identify gaps within the literature.
\end{abstract}

\section{Keywords}

Interventions; Drowning Prevention; Best-Practice; Systematic Review Protocol; Children; Evaluation

\footnotetext{
${ }^{*}$ Corresponding author.
} 


\section{Background}

Globally, the impact of drowning and non-fatal drowning is significant. Drowning continues to be a preventable cause of death worldwide and in 2011, was the greatest (over 22\%) cause of death from unintended injury in children aged less than 14 years with an estimated 129,553 deaths worldwide [1]. These approximations of global drowning deaths are likely to be underreported as many countries provide incomplete or unreliable data. Most drowning deaths in low and middle income countries (LMICs) go unreported, with reporting criteria specifically excluding drowning from suicide, natural disasters (such as floods and tsunamis) and transport/boating incidents [1]-[4]. Age is a major risk factor for drowning, with children aged less than 5 and males aged between 15 - 19 years at heightened risk [5]. Those aged less than 5 are at risk due to a lack of swimming capabilities, an absence of physical barriers, and a lack of close supervision, while those in the teenage years are vulnerable due to alcohol use and increased risk taking behavior [6].

High income countries (HICs) have implemented comprehensive interventions to prevent children from drowning, reducing its occurrence in this age group. However, LMIC have not seen such reductions, as reflected in the injury statistics whereby LMICs experience 96\% of global unintentional drowning deaths [1]. Children in LMICs are at a higher risk of injury than those in HICs, with one quarter of child deaths aged one to four years in the Asia region attributed to drowning, compared to less than $0.01 \%$ in this age group in the Australian population [6]-[8].

Unintentional drowning deaths can be largely averted through a combination of structural, environmental, educational, behavioral and technological interventions [9] [10]. Over the past 50 years, HICs such as Australia, New Zealand and the United Kingdom have achieved a reduction in child drowning through a combination of these approaches [11] [12]. These include the enforcement of residential pool fencing, large scale media campaigns and a continued focus on education and training [13]. This has resulted in improved skills in and around the water via use of: pool and beach lifeguards; development of swimming programs and training to improve the skills of first responders [14] [15]. For many LMICs, a lack of infrastructure and an absence of synergy in the public health system have contributed to slower uptake of interventions [16] though some successes have been achieved through initiatives that include the construction of swimming pontoons and the use of community crèches [17] [18]. Interventions which are successful in HICs are not always feasible in LMICs, and so mechanisms to prevent and reduce the impact of drowning in these different settings warrant further investigation.

Recent systematic reviews on adult public health related drowning interventions [18] have identified a gap in relevant and scientifically sound research in the child drowning literature. For example, Purnell \& McNoe [18] found a lack of international research and practice in the area of alcohol-related water safety policies, and alcohol use and risk taking behaviors of adolescents were identified as leading and potentially modifiable risk factors.

Currently, there is no available systematic review of child public health related drowning interventions. Further, there is insufficient information regarding evaluated and effective interventions to guide the design and delivery of future interventions. Additionally, while evidence of health promotion intervention effectiveness grows, often reviews of the literature do not include details of implementation processes to provide sound evidence for practitioners and policymakers [17]. Furthermore, the difficulty in understanding the causal relationship between public health initiatives and health promotion intervention outcomes is frequently reported as posing conceptual and methodological challenges to the use of results in guiding practice [19] [20]. This lack of process and evidence has practical implications for service delivery organizations, with the potential for a significant gap between the best and actual practice.

Approaches to evaluation of injury prevention and other health promotion initiatives can vary widely and lack methodological rigor [20]. It is frequently reported in the literature that evaluations of injury and/or drowning prevention interventions are limited, being more often used to evaluate short-term changes in knowledge rather than distal outcomes such as behavior change, and that further research is required [5] [21]-[23]. Incorporating systematic evaluation indicators at each stage of program development and implementation would facilitate improved understanding of intervention-outcome links [19]. Use of evaluation frameworks and theory-based interventions can help to address these issues [20] [24]-[26].

Public health plays a key role in developing, monitoring and evaluating public health-related drowning interventions. A comprehensive and systematic approach is required to improve the reporting of interventions to reduce child drowning deaths. This will ensure the use of the best practice, encompassing appropriate theo- 
ries/models and the inclusion of evaluation frameworks. This study aims to assess the global scope of child drowning prevention strategies. This review will investigate the differing designs, settings and effectiveness of prevention interventions associated with drowning in children, substantially adding to the evidence base.

\section{Methods}

The review will be conducted following established criteria for the evidence-based implementation and reporting of systematic reviews using the Preferred Reporting Items for Systematic Reviews and Meta-Analyses (PRISMA) [27]. PRISMA will be used to identify suitable studies for inclusion in the review and to provide transparency and consistency between researchers. The review will follow the same procedures used for a systematic review developed by the WA Centre for Health Promotion Research, Curtin University, Western Australia. To ensure that the quality of implementation and reporting methods outlined in this review are carefully followed, the review has been registered with the PROSPERO International Prospective Register of Systematic Reviews (Registration number: CRD42013004984).

\section{Objectives}

The overall aim of this review is to systematically identify, assess and report on drowning prevention interventions for children (aged 0 to 18 years) that have been published in peer-reviewed literature. This project has two additional objectives: 1) To investigate the utilisation of behaviour change theories/models and evaluation frameworks in guiding child drowning prevention interventions; and 2) To establish which child drowning prevention intervention strategies and methods demonstrate effectiveness in HICs and LMICs.

\section{Criteria for Considering Studies for This Review}

The studies included in the review will be those found in the peer reviewed literature published in English, describing drowning prevention interventions for children aged 0 - 18 years. Interventions developed at an individual, community and population level will be included. Interventions that constitute primary prevention (eliminating or reducing causes of poor health, promotion of protective factors) will be included [28]. Secondary and tertiary interventions (early detection, delaying complications, management and rehabilitation) that occur after the possibility of prevention, such as resuscitation, will be excluded [28]. For the purposes of this review, the definition of drowning from the World Health Organization (WHO) has been used which states that "Drowning is the process of experiencing respiratory impairment from submersion/immersion in liquid; outcomes are classified as death, morbidity and no morbidity" [1].

\subsection{Types of Studies}

The inclusion of a range of intervention study designs is important to assess the breadth of literature available. For the purposes of this review, this will include randomized and non-randomized controlled trials, cohort studies, case-control studies, cross-sectional studies, retrospective analysis, observational studies and qualitative studies.

\subsection{Study Setting and Participants}

The review will assess public health related drowning interventions on an international scope. Studies will include participants aged from birth to those aged 18. Eighteen years of age has been designated as the age at which childhood ends by the UNICEF Convention on the Rights of the Child [29]. Exceptions will be made, to increase or decrease the interval age, in order to match the ages some countries consider to be a cut off for adulthood. Interventions that aim to impact on both children aged less than 18 years and adults aged over 18 years will be included when there is clear reporting on the impact of the intervention on children.

\subsection{Outcome Measures}

Outcome measures will include drowning rates, water safety behavior change or change in behavioral intention, drowning awareness/knowledge/intention/attitude change, water safety policy and legislation, changes to environment and water safety skills. 


\subsection{Search Strategy}

An electronic search will be conducted through the following academic databases to identify relevant publications which describe interventions to prevent infant, child and adolescent drowning deaths worldwide: PubMed; JSTOR; CINAHL; EMBASE; ERIC; Proquest; PsycINFO; Science Direct; Scopus; Global Health; Web of Science; Current Contents; Wiley Online Library and Medline.

Databases searches will be date-limited to before May 31 2013. Searched fields will be keyword, title and abstract. Two independent researchers will complete the following key word searches in the selected databases:

1) infant OR child* OR adolescen* OR kid* OR boy* OR girl* OR young OR youth OR teen*

2) drown* OR submers* OR "water safety"

3) injur* OR mortality OR morbidity

4) interven* OR evaluat* OR "best practice" OR "good practice" OR "best practise” OR "good practise" "health promot*" OR "public health" OR polic* OR research* OR prevent*

The researchers will cross check 10 per cent of search outcomes to ensure consistency in meeting the set criteria. Researchers will review the reference lists of journals articles published in 2012 to May 312013 to assess whether database results are exhaustive. Additionally they will manually search records from the last two years published in the five most common journals established by the electronic searches [30]. Broad search terms will be used on selected databases which will yield a higher number of results. Google Scholar will also be used to cross check articles in the Endnote library after all searching of individual databases is complete.

\subsection{Screening}

Citation management software will be used to manage articles. All citations will be downloaded into Endnote X6 software. Duplicates will be removed after search results have been imported in to the software at which point screening will commence. Screening will be piloted with the research team using criteria established in previous reviews. Titles and abstracts will be screened for relevance using the inclusion criteria. Publications that do not meet the inclusion criteria will be identified by titles and abstracts, which will be checked by two reviewers. Next, the remaining publications will be screened based on their full text and assessed as to whether they meet the stated inclusion criteria and checked by the research team. Citations will be assessed and categorised into three groups: possibly relevant studies; background literature and excluded studies. The full-text of any studies deemed possibly suitable for inclusion will be retrieved. These will be then reviewed to decide eligibility against the inclusion criteria.

\subsection{Quality Appraisal}

Quality appraisal will assess the methodological quality of included studies and then determine whether a study has addressed the likelihood of bias in its design, conduct and analysis [31]. Quality appraisal will be independently completed by two researchers using a checklist adapted for use from the National Institute for Health and Care Excellence (NICE) Quality Appraisal Checklist [32] and the Joanna Briggs series of assessment and review instruments [33]. FL and LN will be the expert reviewers and will perform the quality appraisal. At the time of writing the review, their credentials will include CEO of the peak body of international and public health related drowning interventions, and a senior public health practitioner in a jurisdictional based lifesaving organisation. Their combined expertise equates to more than 25 years of experience in public health design, delivery and evaluation of public health interventions to prevent non-fatal drowning. Discrepancies will be resolved by discussion between reviewers. Any arbitration will be resolved by consensus within the research team.

\subsection{Data Extraction and Management}

Data extraction will identify and record descriptive and outcome data for each article that is included in the review. Extraction will be independently completed by two researchers using a standardised data extraction form [32] [33]. This will ensure researchers are consistent in their extraction processes, minimising bias and facilitating the accurate presentation of the data. Discrepancies will be resolved as described above. Data entered will be cross-checked to ensure that there are no data entry errors. 


\subsection{Analysis, Synthesis and Reporting}

Data analysis and presentation of the results will occur after data has been extracted. A descriptive evaluation of each study will occur which will be presented in a table. It is not expected that there will be any homogeneity within the data and therefore meta-analysis and synthesis will be not be undertaken. Basic reporting of "pooled" data on age, gender, and key findings will be included. SPSS software, version 19 [34] will be utilised for any statistical calculations of descriptives. The results will lead to conclusions and recommendations regarding future health promotion or research presented in a narrative review. Once the data has been synthesised, initial findings will be presented to relevant non-government and government stakeholders to facilitate the translation of the findings into policy and practice.

\section{Discussion}

Anecdotally, practitioners indicate that the peer reviewed literature lacks sufficient and robust policy-ready evidence on the effectiveness of interventions to prevent child drowning. Results of evaluation must be reported, documented and shared so that intervention effectiveness can inform best practice and prioritisation of interventions [3]. This review will provide an opportunity to map the types of interventions being implemented worldwide to prevent drowning amongst children, highlight gaps in the literature, and determine the most effective interventions in different settings. It is anticipated that the research will be disseminated via national and international academic and/or practitioner conferences. A summary will be disseminated to relevant stakeholders that will present key findings and implications for policy and practice, and the resulting compiled data will be collated and submitted to a peer-reviewed journal for wider dissemination.

\section{Conclusion}

This review of international child drowning prevention will not only add to the small evidence base of effective implementation and evaluation of child drowning prevention interventions, but may also support the development of a best practice model. The review will function as a call to action for practitioners to systematically report on interventions that work and more importantly those that do not to create a critical mass of research and evaluation on drowning within the peer-reviewed literature. This review will provide the most recent and comprehensive available evidence to catalogue what data exist, and further, what works to reduce the burden of child drowning globally.

\section{Acknowledgements}

There is no conflict of interest of any parties contributing to this study.

\section{References}

[1] World Health Organization (2012) Drowning. http://www.who.int/mediacentre/factsheets/fs347/en/

[2] International Life Saving Federation (2007) World Drowning Report. International Life Saving Federation, Belgium.

[3] International Life Saving Federation (2008) Position Statement: ILS Risk Assessment Framework. International Life Saving Federation, Belgium.

[4] Smith, G. (2006) The Global Burden of Drowning. In: The Handbook of Drowning, 56-61.

[5] Organisation, W.H. (2008) World Report on Child Injury Prevention. World Health Organisation, Geneva.

[6] Royal Life Saving Society Australia (2012) Royal Life Saving Society_Australian National Drowning Report 2012. Royal Life Saving Society Australia, Sydney.

[7] Plitponkarnpim, A., Anderson, R., Jansson, B., et al. (1999) Unintentional Injury Mortality in Children: A Priority for Middle Income Countries in the Advanced Stage of Epidemiological Transition. Injury Prevention, 5, 98-103. http://dx.doi.org/10.1136/ip.5.2.98

[8] Linnan, M., Rahman, A., Scarr, J., et al. (2012) Child Drowning: Evidence for a Newly Recognized Cause of Child Mortality in Low and Middle Income Countries in Asia. UNICEF Office of Research, Florence.

[9] Ashby, K., Routley, V. and Stathakis, V. (1998) Enforcing Legislative and Regulatory Injury Prevention Strategies. Hazard, 1-16.

[10] Paine, N. and Cassell, E. (2003) Local Government Enforcement of Private Swimming Pool Safety Regulations- 
Survey of Council Building Surveyors/Inspectors. Hazard, 2-20.

[11] Weiss, J. (2010) Prevention of Drowning. Pediatrics, 126, e253-e262. http://dx.doi.org/10.1542/peds.2010-1265

[12] Australian Water Safety Council (2012) Australian Water Safety Strategy 2012-2015. Australian Water Safety Council, Sydney.

[13] Thompson, D. and Rivara, F. (1998) Pool Fencing for Preventing Drowning of Children. Cochrane Database of Systematic Reviews, 1, 1-13.

[14] Brenner, R., Saluja, G. and Smith, G. (2003) Swimming Lessons, Swimming Ability, and the Risk of Drowning. Injury Control and Safety Promotion, 10, 211-215. http://dx.doi.org/10.1076/icsp.10.4.211.16775

[15] Bugeja, L. and Franklin, R. (2012) An Analysis of Stratagems to Reduce Drowning Deaths of Young Children in Private Swimming Pools and Spas in Victoria, Australia. International Journal of Injury Control and Safety Promotion, 20, 282-294. http://dx.doi.org/10.1080/17457300.2012.717086

[16] Bartlett, S. (2002) The Problem of Children's Injuries in Low-Income Countries: A Review. Health Policy and Planning, 17, 1-13. http://dx.doi.org/10.1093/heapol/17.1.1

[17] Roen, K., Arai, L., Roberts, H., et al. (2006) Extending Systematic Reviews to Include Evidence on Implementation: Methodological Work on a Review of Community-Based Initiatives to Prevent Injuries. Social Science and Medicine, 63, 1060-1071. http://dx.doi.org/10.1016/j.socscimed.2006.02.013

[18] Purnell, M. and McNoe, B. (2008) Systematic Review of Drowning Interventions and Risk Factors and International Comparison of Water Safety Policies and Programs: Report to the Accident Compensation Corporation. Injury Prevention Research Unit, University of Otago, Dunedin.

[19] Davies, J. and Sherriff, N. (2011) The Gradient in Health Inequalities among Families and Children: A Review of Evaluation Frameworks. Health Policy, 101, 1-10. http://dx.doi.org/10.1016/j.healthpol.2010.09.015

[20] Levine, E., Abbatangelo-Gray, J., Mobely, A., et al. (2012) Evaluating MyPlate: An Expanded Framework Using Traditional and Nontraditional Metrics for Assessing Health Communication Campaigns. Journal of Nutrition Education and Behavior, 44, s2-s12. http://dx.doi.org/10.1016/j.jneb.2012.05.011

[21] Bristow, K., Carson, J.B., Warda, L., et al. (2002) Childhood Drowning in Manitoba: A 10-Year Review of Provincial Paediatric Death Review Committee Data. Paediatrics \& Child Health, 7, 637-641.

[22] Franklin, R. and Pearn, J.H. (2010) Drowning for Love: The Aquatic Victim-Instead-of-Rescuer Syndrome: Drowning Fatalities Involving Those Attempting to Rescue a Child. Journal of Paediatrics and Child Health, 47, 44-47. http://dx.doi.org/10.1111/j.1440-1754.2010.01889.x

[23] Hyder, A., Borse, N.N., Blum, L., et al. (2008) Childhood Drowning in Low- and Middle-Income Countries: Urgent Need for Intervention Trials. Journal of Paediatrics and Child Health, 44, 221-227. http://dx.doi.org/10.1111/j.1440-1754.2007.01273.x

[24] Polinder, S., Segui-Gomez, M., Toet, H., et al. (2012) Systematic Review and Quality Assessment of Economic Evaluation Studies of Injury Prevention. Accident Analysis and Prevention, 45, 211-221. http://dx.doi.org/10.1016/j.aap.2011.07.004

[25] Gregson, J., Foerster, S.B., Orr, R., et al. (2001) System, Environmental, and Policy Changes: Using the Social- Ecological Model as a Framework for Evaluating Nutrition Education and Social Marketing Programs with Low-Income Audiences. Journal of Nutrition Education, 33, s4-s15. http://dx.doi.org/10.1016/S1499-4046(06)60065-1

[26] Medeiros, L.C., Butkus, S.N., Chipman, H., et al. (2005) A Logic Model Framework for Community Nutrition Education. Journal of Nutrition Education and Behavior, 37, 197-202. http://dx.doi.org/10.1016/S1499-4046(06)60246-7

[27] Moher, D., Liberati, A., Tetzlaff, J., et al. (2009) Preferred Reporting Items for Systematic Reviews and Meta-Analyses: The PRISMA Statement. PLOS Medicine, 6, e1000097. http://dx.doi.org/10.1371/journal.pmed.1000097

[28] NPH (2004) The National Injury Prevention and Safety Promotion Plan: 2004-2014. National Public Health Partnership, Canberra.

[29] UNICEF (1989) Convention on the Rights of the Child. UNICEF, Florence. http://www.unicef-irc.org/portfolios/crc.html

[30] Bambra, C.L., Hillier, F.C., Moore, H.J., et al. (2013) Tackling Inequalities in Obesity: A Protocol for a Systematic Review of the Effectiveness of Public Health Interventions at Reducing Socioeconomic Inequalities in Obesity among Adults. Journal of Systematic Reviews, 2, 1-17.

[31] Higgins, J. and Green, S. (2006) Cochrane Handbook for Systematic Reviews of Interventions 4.2.6. The Cochrane Collaboration.

[32] NIfHaCE (2009) The Guidelines Manual. National Institute for Health and Clinical Excellence, London.

[33] Joanna Briggs Institute (2008) Joanna Briggs Institute Reviewers’ Manual: 2008 Edition. Joanna Briggs Institute, 
G. Crawford et al.

Adelaide.

[34] IBM (2010) SPSS Statistics. SPSS Inc., Chicago. 